\title{
It's Not What You Draw, It's What You Don't Draw
}

\author{
Drawing Paths with Holes in R Graphics \\ by Paul Murrell
}

\begin{abstract}
The $R$ graphics engine has new support for drawing complex paths via the functions polypath() and grid.path(). This article explains what is meant by a complex path and demonstrates the usefulness of complex paths in drawing non-trivial shapes, logos, customised data symbols, and maps.
\end{abstract}

One of the design goals of the $\mathrm{R}$ graphics system is to allow fine control over the small details of plots. One way that the $\mathrm{R}$ graphics system does this is by providing access to low-level generic graphics facilities, such as the ability to draw basic shapes and the ability to control apparently esoteric, but still useful, features of those shapes, such as the line end style used for drawing lines.

In $\mathrm{R}$ version 2.12.0, another low-level graphics facility was added to R: the ability to draw complex paths (not just polygons).

This article describes this new facility and presents some examples that show how complex paths might be useful.

\section{Drawing paths with holes}

The concept of a path is similar to the concept of a polygon: a path is defined by a series of $(x, y)$ locations that describe the boundary of the path.

For example, the following code defines a set of $(x, y)$ locations that describe a simple triangle.

$>\mathrm{x}<-\mathrm{C}(.1, .5, .9)$

$>\mathrm{y}<-\mathrm{c}(.1, .8, .1)$

A triangle can be drawn from these locations using either the polypath () function from the graphics package or, as shown below and in Figure 1, using the grid.path () function from the grid package.

$>$ library (grid)

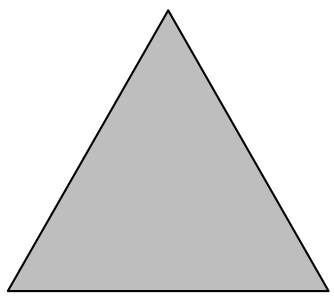

Figure 1: A triangle drawn by the grid.path () function from a set of three $(x, y)$ locations.

As for any basic shape, it is possible to control the colour and thickness of the path border and the colour used to fill the interior of the path.

We can also provide more than one set of $(x, y)$ locations when drawing a path. The following code provides an example, defining a new set of six locations along with an id vector that can be used to break the locations into two groups of three.

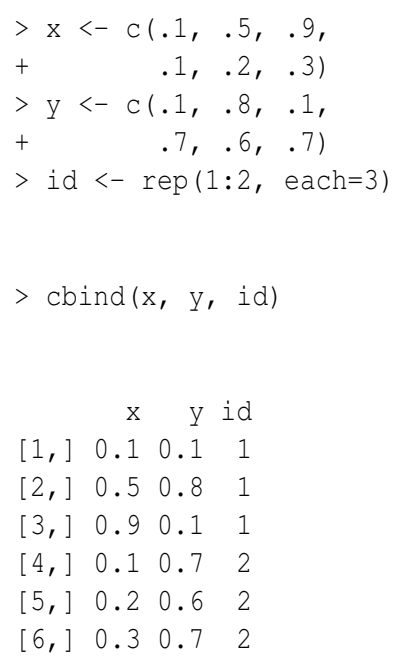

$[1] \quad 0.1 \quad 0.1 \quad$,

$[2] \quad 0.5 \quad 0.8 \quad$,

$[3] \quad 0.9 \quad 0.1 \quad$,

$\left[\begin{array}{llll}4,] & 0.1 & 0.7 & 2\end{array}\right.$

$\left[\begin{array}{lllll}5,] & 0.2 & 0.6 & 2\end{array}\right.$

$\left[\begin{array}{lllll}6,] & 0.3 & 0.7 & 2\end{array}\right.$

These locations can be used to describe a path that consists of two distinct triangles. The following code draws such a path using grid.path(). The id argument is used to identify distinct groups of locations when using grid.path (). ${ }^{1}$ Figure 2 shows the result of drawing this path.

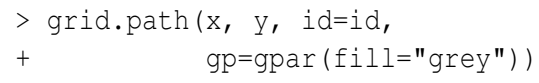

\footnotetext{
${ }^{1}$ When using polypath (), NA values must be inserted between distinct groups of $(x, y)$ values.
} 


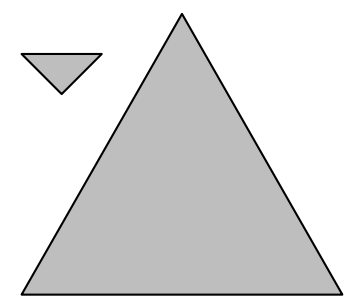

Figure 2: Two triangles drawn by the grid.path() function from a set of six $(x, y)$ locations broken into two groups of three locations.

This output looks exactly the same as the output we would get from drawing the two groups of locations as polygons, using grid.polygon() or polygon(), but conceptually there is a difference because the path treats the two groups of locations as defining a single shape. We can see the difference more clearly if we move the smaller triangle so that it lies within the larger triangle (see Figure 3).
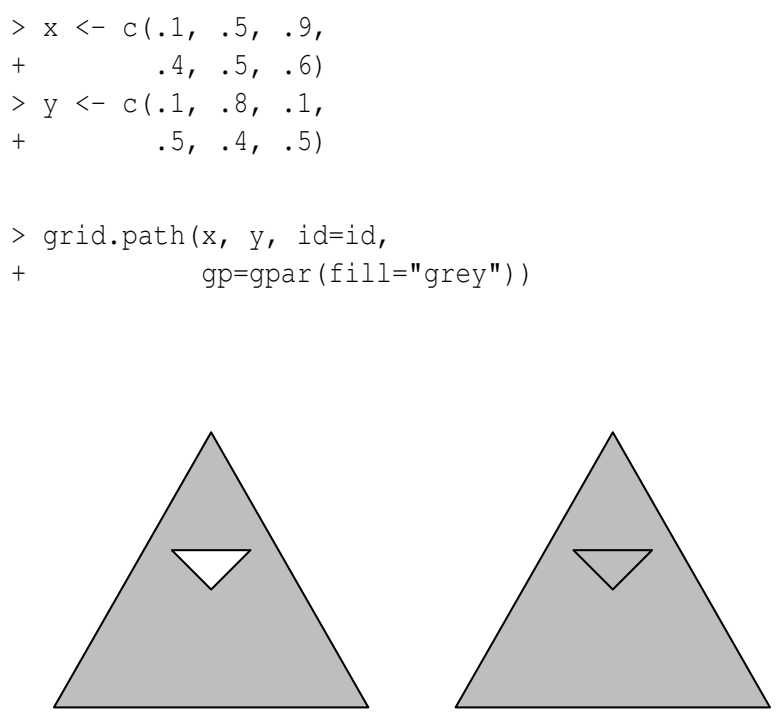

Figure 3: On the left is a path drawn by the grid.path() function where the boundary of the path consists of two distinct triangles (one within the other). The result is a single shape with a hole in it. On the right is the result of drawing the two boundaries with the grid.polygon () function, which treats the boundaries as two separate shapes. In this case, the smaller triangle is drawn (filled in) on top of the larger triangle.

This example demonstrates that the two triangles together define a single shape, which is a triangular region with a triangular hole in it. The interior of the shape-the area that is shaded-does not include the region within the smaller triangle.

\section{Fill rules}

There are two ways to determine the interior of a path like this. The default is called the non-zero winding rule. This draws an imaginary straight line and looks at where the straight line intersects the boundary of the shape. A count is made of how many times the boundary is running left-to-right at the intersection and how many times the boundary is running right-to-left; if the two counts are the same then we are outside the shape and if the counts are different, we are inside the shape.

To see this more clearly, Figure 4 uses lines with arrows to show the directions on the boundaries of the path from Figure 3.

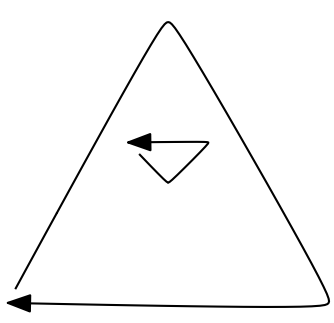

Figure 4: The direction of the boundaries for the path in Figure 3.

The outer triangle boundary is clockwise and the inner triangle boundary is anti-clockwise, so, using the non-zero winding rule, the region within the inner triangle is actually outside the path. A straight line from inside the inner triangle to outside the outer triangle intersects two boundaries, one going right-to-left and one going left-to-right.

To further demonstrate this rule, the following code defines a more complex path, this time consisting of three triangles: one large clockwise triangle, with two smaller triangles inside, one clockwise and one anti-clockwise.

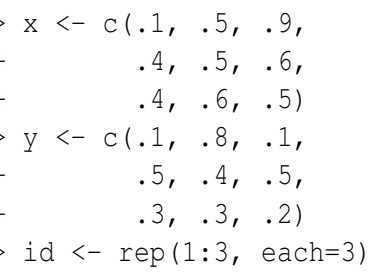

Figure 5 shows a diagram of the boundary directions and the result of drawing this path. Because the second smaller triangle is clockwise, the region inside that triangle is still part of the interior of the path, according to the non-zero winding rule.

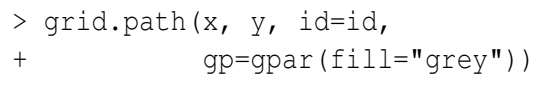



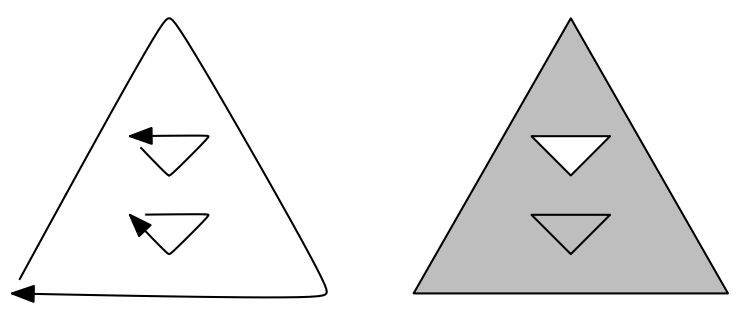

Figure 5: A path where the boundary consists of three triangles (two smaller ones within one larger one). The diagram on the left shows the direction of the boundaries for the path. On the right, the path is drawn by the grid.path () function, with the interior of the path determined using the non-zero winding rule.

The other rule for determining the interior of a path is called the even-odd rule. This just draws an imaginary straight line through the shape and counts how many times the straight line crosses the boundary of the shape. Each time a boundary is crossed, we toggle between outside and inside the shape.

The following code draws the same path as in Figure 5, but uses the even-odd rule to determine the shape's interior. This time, the result is a larger triangle with two smaller triangular holes punched out of it (see Figure 6).
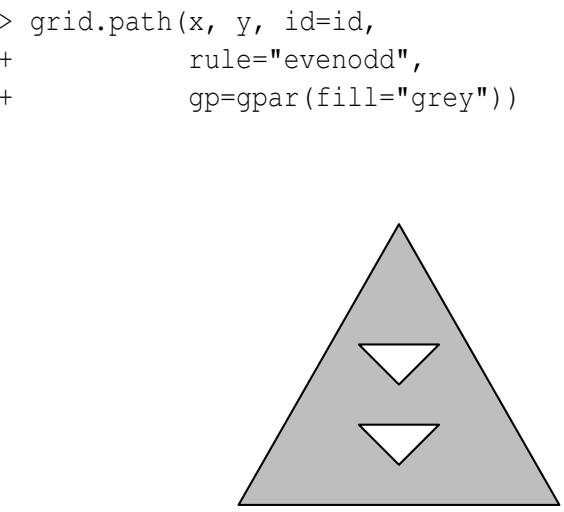

Figure 6: The path from Figure 5 drawn using the even-odd fill rule.

The SVG language specification contains a nice simple explanation and demonstration of these fill rules; see http://www.w3.org/TR/SVG/painting. html\#FillRuleProperty.

\section{Applications}

So what can these complex paths be used for? The possibilities are endless, but this section describes a couple of concrete examples. The $\mathrm{R}$ code for these examples can be obtained from the online resources that accompany this article. ${ }^{2}$

A trivial observation is that complex paths allow us to draw complex shapes. The triangle with triangular holes from the previous section is an example of a complex shape; it is not possible to describe this shape as a simple polygon.

Another way that paths can be useful for drawing complex shapes is that they allow us to combine several simpler shapes to construct a more complex whole. Figure 7 shows an example of this, where the overall shape shape has a very complex outline, but it can be constructed as a path simply by combining circles and triangles.
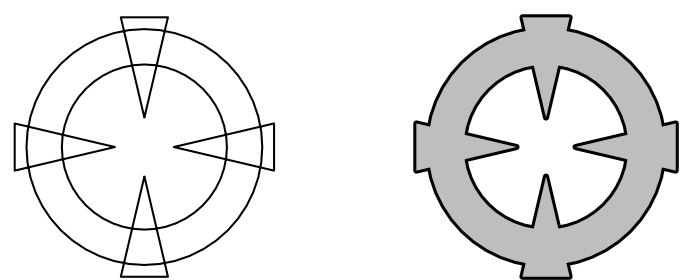

Figure 7: A complex shape constructed from simple shapes combined together to make a path.

Figure 8 shows how this shape might be used to dramatically highlight a point of interest within a graph (in this case, to bring attention to the data for the Ferrari Dino in the mtcars data set).

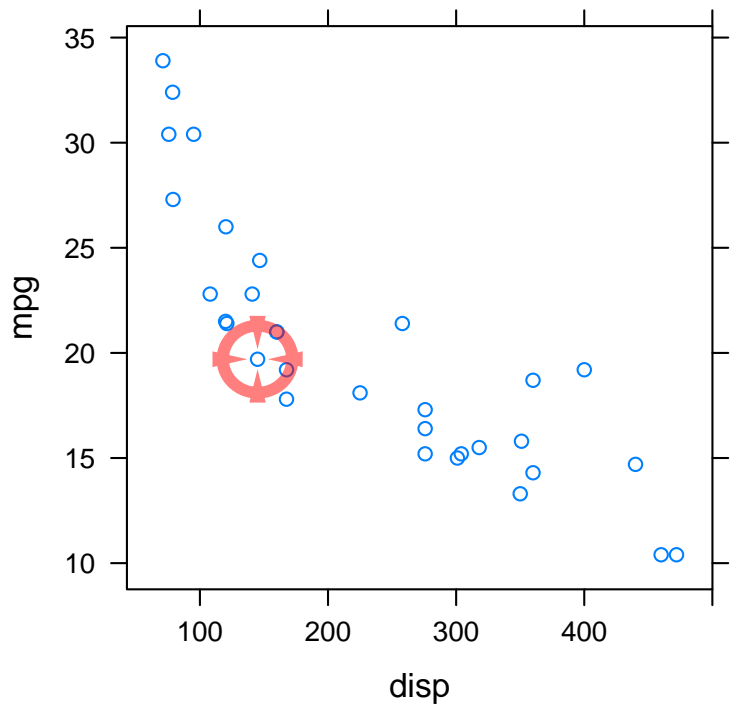

Figure 8: A plot with a complex path used to highlight a special point of interest.

Another situation where the ability to draw com- 
plex paths can be useful is if we are trying to draw a shape that someone else has created. For example, we might want to draw the logo of a company or an organisation as a label on a plot.

Figure 9 shows the GNU logo. This image consists of a single complex path, so we must be able to draw such paths in order to render it correctly.
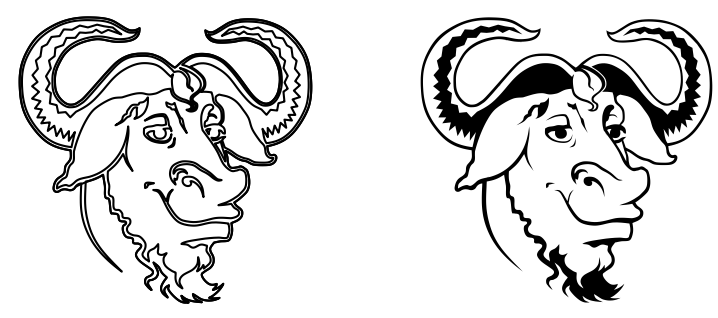

Figure 9: A complex path that describes the GNU logo.

Figure 10 shows the GNU logo being used as a background watermark for a lattice barchart (Sarkar, 2008).

\section{Number of Citations per Year}

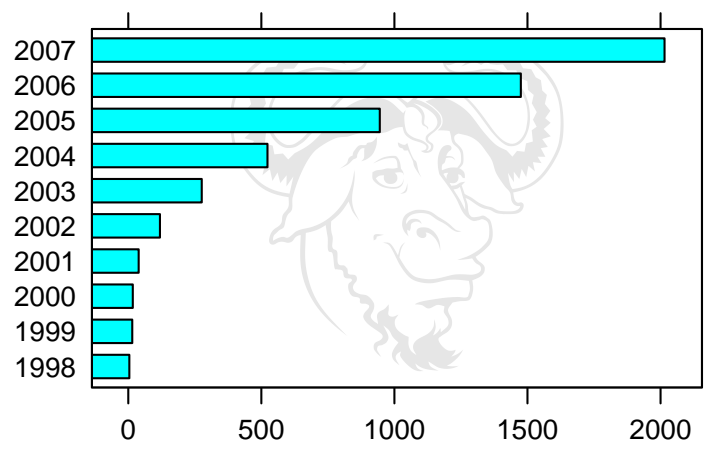

Figure 10: A plot with the GNU logo from Figure 9 as a background watermark.

Another way that we might use external complex shapes is as data symbols on a plot. Figure 11 shows a bus icon. Again, this bus icon is a single path so it must be drawn using grid.path () or polypath() in order for it to render correctly.
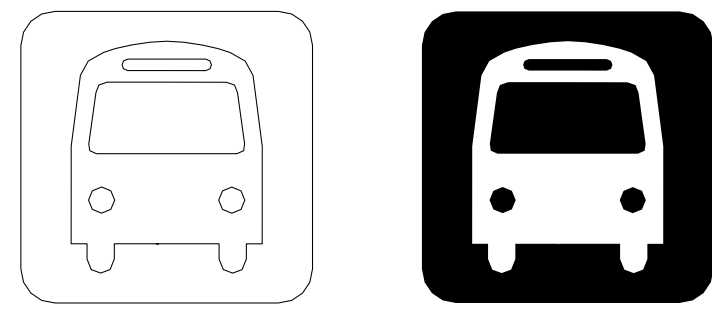

Figure 11: A path that describes a bus icon.

Figure 12 shows this bus icon being used as data symbols on a lattice scatterplot of daily bus ridership data.

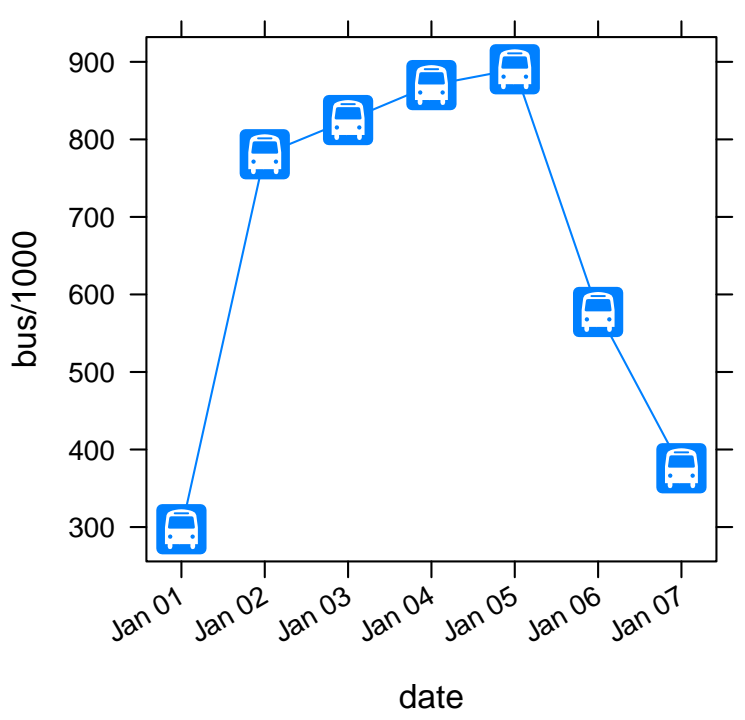

Figure 12: A plot with the bus icon from Figure 11 used as a data symbol.

Another general area where complex paths arise is the drawing of maps. The outline of a country's borders or coastline represents a set of $(x, y)$ coordinates, often in a very complicated arrangement. One situation where it can be useful to treat the map outline as a path is the case where a country contains a lake; the lake can be thought of as a hole in the country shape. Things can get quite complicated if the lake then contains an island, and the island has a lake, and so on. If the map is treated as a path to fill then all of these cases are dealt with quite easily.

Figure 13 shows a map of part of the South Island of New Zealand. The lake in the lower right quadrant of this map is Lake Te Anau and at the base of one of the westerly spurs of this lake is an island. This map outline has been drawn as a path with a green fill colour used to indicate land area and an appropriate fill rule ensures that the lake is not filled in, but the island on the lake is. 


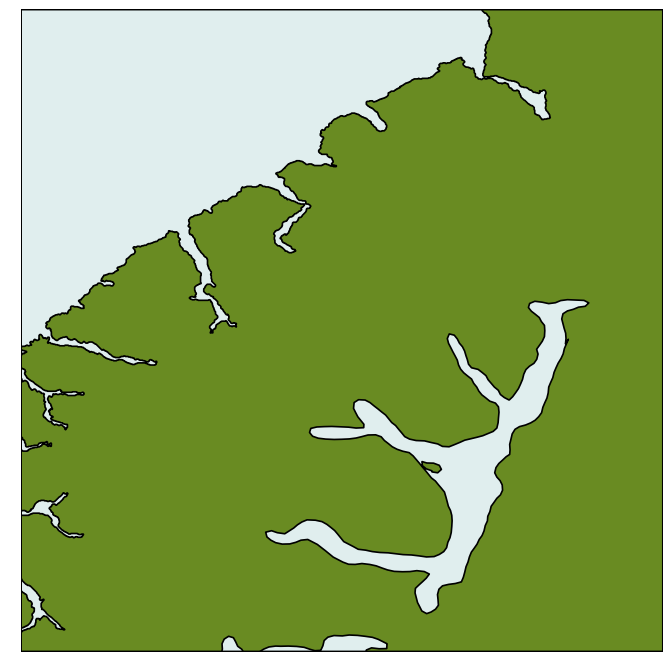

Figure 13: A map showing an island in a lake on an island.

Although R provides many low-level graphics facilities, such as the ability to draw complex paths, there are still some basic tricks that it does not yet support. One example is the ability to clip output to an arbitrary region on the page (it is currently only possible to clip to rectangular regions with $\mathrm{R}$ ).

Sometimes, a missing feature like this can be worked around by making inventive use of the existing facilities. Figure 14 shows an example of this, where a contour of earthquake events has been overlaid on a map of New Zealand, but the contours are only visible over land areas.

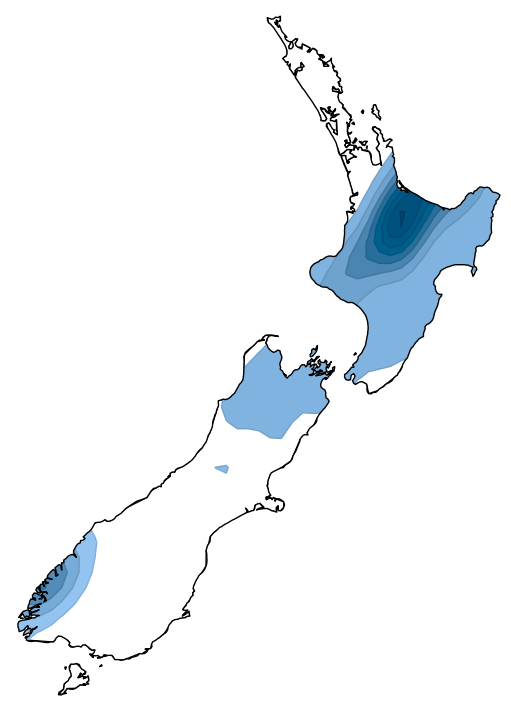

Figure 14: A map with an overlaid contour. A path has been used to obscure the contour where it does not overlap with land.

This result was achieved using complex paths (see Figure 15). The starting point is the entire con- tour overlaid on a New Zealand map (left). A path is constructed from the New Zealand coastline (middle), and then a bounding rectangle is added to the path (right). This combined path allows us to fill a region that is everything outside of the New Zealand coastline and that can be drawn on top of the original image to obscure those parts of the contour that are not over land.

\section{Caveats}

The polypath() and grid.path() functions are only supported on the pdf(), postscript(), x11 (type="cairo"), windows(), and quartz() graphics devices (and associated raster formats).

These functions are not supported on $\mathrm{x} 11$ (type="Xlib"), xfig(), or pictex () and support is not guaranteed on graphics devices provided by extension packages.

\section{Summary}

There are new functions, polypath() and grid.path() for drawing complex paths, including paths with holes, in R graphics output. These functions can be useful for drawing non-trivial shapes, logos, custom data symbols, and maps.

\section{Acknowledgements}

The following material and data were used in this article:

- The GNU logo was created by Aurelio A. Heckert and is available from http: / /www.gnu.org/ graphics/heckert_gnu.html.

- The bus icon was created by the Geographics Unit, School of Environment, The University of Auckland.

- Both the GNU logo and the bus icon shape information were imported into $\mathrm{R}$ and drawn using the grImport package (Murrell, 2009).

- The bus data were obtained from the City of Chicago Data Portal http:// data.cityofchicago.org/Transportation/ CTA-Ridership-Daily-Boarding-Totals/.

- The detailed map of the South Island of New Zealand came from the Global Selfconsistent, Hierarchical, High-resolution Shoreline Database (version 2.0; Wessel and Smith, 1996) and was loaded into $R$ using the maptools package (Lewin-Koh and Bivand, 2011).

- The earthquake data came from the GeoNet Project: http: //www.geonet.org.nz/ 

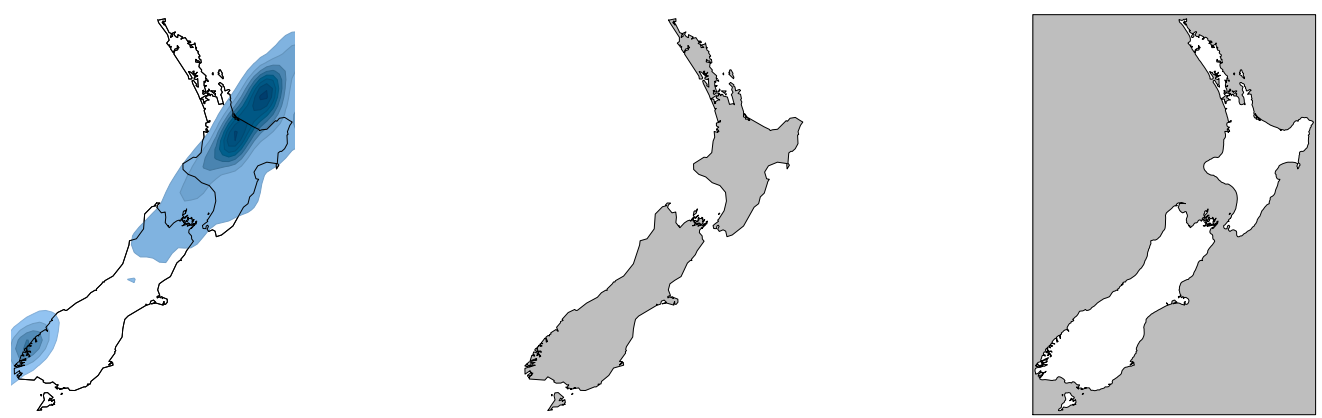

Figure 15: A map with a path used to obscure unwanted drawing.

- The New Zealand coastline information for Figures 14 and 15 came from the maps package (Brownrigg and Minka, 2011).

Many thanks also to the anonymous reviewers who suggested several useful improvements to this article.

\section{Bibliography}

R. Brownrigg and T. P. Minka. maps: Draw Geographical Maps, 2011. URL http: / / CRAN . R-pro ject .org/ package=maps. $\mathrm{R}$ package version 2.1-6. [p18]

N. J. Lewin-Koh and R. Bivand. maptools: Tools for reading and handling spatial objects, 2011 . URL http: //CRAN.R-project.org/package=maptools. R package version 0.8-6. [p17]

P. Murrell. Importing vector graphics: The grImport package for R. Journal of Statistical Software, 30(4): 1-37, 2009. URL http: //www. jstat soft.org/v30/ i $04 /$. [p17]

D. Sarkar. Lattice: Multivariate Data Visualization with R. Springer, New York, 2008. URL http: / / lmdvr. r-forge.r-project.org. ISBN 978-0-387-75968-5. [p16]

P. Wessel and W. H. F. Smith. A global self-consistent, hierarchical, high-resolution shoreline database. Journal of Geophysical Research, pages 8741-8743, 1996. [p17]

Paul Murrell

Department of Statistics

The University of Auckland

New Zealand

paulestat.auckland.ac.nz 